\title{
New delivery systems for amphotericin B applied to the improvement of leishmaniasis treatment
}

\author{
Miguel Angel Chávez-Fumagalli ${ }^{[1]}$, Tatiana Gomes Ribeiro ${ }^{[2]}$, Rachel Oliveira Castilho ${ }^{[2][3]}$, \\ Simone Odília Antunes Fernandes ${ }^{[4]}$, Valbert Nascimento Cardoso ${ }^{[4]}$, \\ Cecília Steinberg Perilo Coelho ${ }^{[5]}$, Débora Vasconcelos Costa Mendonça ${ }^{[6]}$, \\ Manuel Soto ${ }^{[7]}$, Carlos Alberto Pereira Tavares ${ }^{[8]}$, André Augusto Gomes Faraco ${ }^{[2],[3]}$ \\ and Eduardo Antonio Ferraz Coelho ${ }^{[1],[6]}$
}

[1]. Programa de Pós-Graduação em Ciências da Saúde: Infectologia e Medicina Tropical, Faculdade de Medicina, Universidade Federal de Minas Gerais, Belo Horizonte, Minas Gerais, Brasil. [2]. Programa de Pós-Graduação em Ciências Farmacêuticas, Faculdade de Farmácia, Universidade Federal de Minas Gerais, Belo Horizonte, Minas Gerais, Brasil. [3]. Departamento de Produtos Farmacêuticos, Faculdade de Farmácia, Universidade Federal de Minas Gerais, Belo Horizonte, Minas Gerais, Brasil. [4]. Departamento de Análises Clínicas e Toxicológicas, Faculdade de Farmácia, Universidade Federal de Minas Gerais, Belo Horizonte, Minas Gerais, Brasil. [5]. Bioprospection Ltda., Belo Horizonte, Minas Gerais, Brasil. [6]. Departamento de Patologia Clínica, Colégio Técnico, Universidade Federal de Minas Gerais, Belo Horizonte, Minas Gerais, Brasil. [7]. Centro de Biología Molecular Severo Ochoa, Consejo Superior de Investigaciones Científicas-Universidad Autonoma de Madrid, Universidad Autónoma de Madrid, Madrid, Espanha. [8]. Departamento de Bioquímica e Imunologia, Instituto de Ciências Biológicas, Universidade Federal de Minas Gerais, Belo Horizonte, Minas Gerais, Brasil.

\begin{abstract}
Leishmaniasis is one of the six major tropical diseases targeted by the World Health Organization. It is a life-threatening disease of medical, social and economic importance in endemic areas. No vaccine is yet available for human use, and chemotherapy presents several problems. Pentavalent antimonials have been the drugs of choice to treat the disease for more than six decades; however, they exhibit high toxicity and are not indicated for children, for pregnant or breastfeeding women or for chronically ill patients. Amphotericin B (AmpB) is a second-line drug, and although it has been increasingly used to treat visceral leishmaniasis (VL), its clinical use has been hampered due to its high toxicity. This review focuses on the development and in vivo usage of new delivery systems for AmpB that aim to decrease its toxicity without altering its therapeutic efficacy. These new formulations, when adjusted with regard to their production costs, may be considered new drug delivery systems that promise to improve the treatment of leishmaniasis, by reducing the side effects and the number of doses while permitting a satisfactory cost-benefit ratio.
\end{abstract}

Keywords: Amphotericin B. Leishmaniasis. Nanoparticles. Toxicity. Treatment. Lipid-based formulations.

\section{INTRODUCTION}

Leishmaniasis presents a broad spectrum of clinical manifestations and is caused by different species of protozoa belonging to the genus Leishmania ${ }^{(1)}$. The disease displays high morbidity and mortality throughout the world, with approximately 350 million people in 98 countries at risk of contracting the infection $^{(2)}$. Approximately 700,000 to 1.2 million cases of tegumentary leishmaniasis (TL) and 200,000 to 400,000 cases of visceral leishmaniasis $(\mathrm{VL})$ are registered annually ${ }^{(3)}$. Visceral leishmaniasis represents an important disease worldwide, leading to nearly 50,000 deaths each year ${ }^{(4)(5)(6)}$. Because of its remarkable impact on global public health, leishmaniasis is considered

Corresponding author: Prof. Eduardo Antonio Ferraz Coelho. Laboratório de Biotecnologia Aplicada ao Estudo das Leishmanioses/Depto. de Patologia Clínica/COLTEC/UfMG. Avenida Antônio Carlos 6627, 31270-901 Belo Horizonte, Minas Gerais, Brasil.

Phone/Fax: 5531 3409-4983

e-mail: eduardoferrazcoelho@yahoo.com.br

Received 24 April 2015

Accepted 29 May 2015 one of the six major tropical diseases by the World Health Organization, and it has gained greater importance in HIVinfected individuals as an opportunistic infection in areas where both infections are endemic ${ }^{(7)}$. Leishmaniasis has thus become the third most common parasitic disease after toxoplasmosis and cryptosporidiosis ${ }^{(8)}$.

In Brazil, VL is caused by Leishmania (Leishmania) infantum chagasi species $^{(9)}$. Dogs are the main domestic reservoirs of the parasites and play a central role in the transmission cycle between humans and sandflies ${ }^{(10)}$. The disease is characterized by clinical manifestations based on irregular fever, weight loss, pallor, splenomegaly, and pancytopenia, and it carries a high risk of mortality in the absence of treatment ${ }^{(11)}$. Nevertheless, the outcomes of infection can vary from asymptomatic and/ or subclinical disease to an acute symptomatic form. The asymptomatic disease is characterized by subjects infected by the parasites but with no apparent impact on their health, whereas the acute disease is characterized by clinical manifestations such as lymphadenopathy, fever, diarrhea, malaise, coughing, mild hepatomegaly and/or splenomegaly, as well as a positive laboratory diagnosis ${ }^{(12)}$. 
Currently, there is no gold-standard test for the diagnosis of leishmaniasis, and a combination of different methods is frequently needed to obtain more precise results. The serological diagnosis of VL has been based on the detection of antileishmanial antibodies in serum samples of patients. However, these tests have problems related to their sensitivity and/or specificity ${ }^{(13)}$. The parasitological diagnosis is based on the demonstration of amastigote forms in collected specimens; however, these tests require invasive procedures for sample collection, which limit their use in large-scale epidemiological studies $^{(14)}$. The detection of Leishmania spp. Deoxyribonucleic acid (DNA) by the polymerase chain reaction technique is also performed, but it is costly ${ }^{(15)}$.

Historically, chemotherapy to treat leishmaniasis has been based on the parenteral administration of pentavalent antimonials ${ }^{(16)}$. These drugs are effective, presenting an efficacy of approximately $90 \%{ }^{(17)}$, but there has been growing evidence of variable efficacy that depends on the species, geographic region, presence of resistant strains, and therapeutic schemes employed ${ }^{(18)(19)(20)}$. Moreover, certain factors, such as the need for parenteral administration for up to 30 days, the high frequency of side effects such as myalgia, arthralgia, anorexia, fever and urticaria, and significant toxicity to the liver, kidneys and spleen of the patient, have contributed to the limited use of these drugs in clinical practice ${ }^{(21)(22)}$. Pentamidine, another antileishmanial drug, is also unsuitable as a first-line treatment due to its toxicity. Clinical results using oral miltefosine have been encouraging; however, this drug has been linked to teratogenicity and should not be prescribed to pregnant women or to those of childbearing age ${ }^{(23)}$.

Drug delivery systems have received considerable attention in the field of drug development. Pharmaceutical technology has been used to control pharmacokinetic properties, such as absorption, distribution, metabolism, and excretion and to improve the efficacy and/or reduce the toxicity of drugg ${ }^{(24)}$. This review examines the development and deployment of technologies to improve the delivery of drugs for treating leishmaniasis. It focuses on recent improvements that have led to reductions in the toxicity of amphotericin B (AmpB) and to improvements in the effectiveness of AmpB in Leishmania spp. infection models.

\section{AMPHOTERICIN B AND ITS DELIVERY SYSTEMS FOR THE TREATMENT OF LEISHMANIASIS}

Drug discovery is typically a long and very expensive process, requiring an average of 10 to 20 years and an investment of more than $\$ 1.0$ billion before a new compound with activity against a specific target is identified, characterized, and developed for clinical applications ${ }^{(25)}{ }^{(26)}$. In the case of leishmaniasis, there are few new antileishmanial drugs in the pipeline, and drug resistance is increasing. Over the past few decades, AmpB (Figure 1) has been used with increasing frequency to treat leishmaniasis. It has been employed to treat systemic fungal infections, and its mechanism of action is related to its binding to the membrane sterols of parasites. Because Leishmania spp. membranes also contain ergosterol, the drug impairs the cell

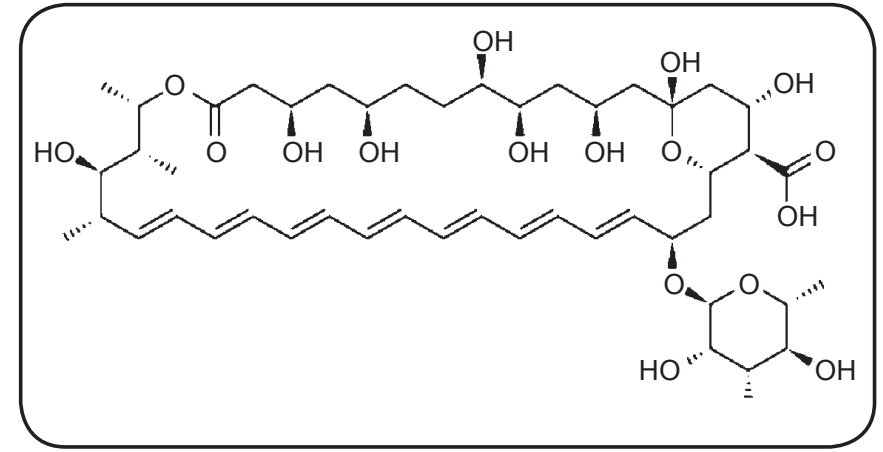

FIGURE 1 - Chemical structure of amphotericin B.

permeability of these parasites, leading to the loss of cations, such as $\mathrm{K}^{+}$and causing cell death ${ }^{(27)}$. Moreover, AmpB shows effective antileishmanial activity against different Leishmania spp. species that have clinical relevance in the Americas, such as L. infantum, L. braziliensis and L. amazonensis ${ }^{(28)}$. However, the clinical use of AmpB has been limited due to its high toxicity, which can cause such clinical symptoms in patients as nephrotoxicity, cardiac alterations, hemolysis and liver damage, as well as nausea and fever ${ }^{(29)(30)}$.

To reduce the toxicity of $\mathrm{AmpB}$, lipid-based formulations have been developed ${ }^{(31)}$. A liposomal formulation (AmBisome $\left.{ }^{\circledR}\right)$, an AmpB colloidal dispersion (Amphocil ${ }^{\circledR}$ ), and an AmpBlipid complex (Abelcet ${ }^{\circledR}$ ) have been used for the treatment of leishmaniasis ${ }^{(32)}$ (Figure 2). The World Health Organization has recommended the use of liposomal AmpB based on its high levels of efficacy and safety ${ }^{(2)}$. The development of these formulations has diminished drug toxicity during the treatment of leishmaniasis ${ }^{(33)}$. In general, these products are less nephrotoxic than free AmpB, as they are taken up selectively by macrophages. Additionally, adverse effects, including mild urticarial rash and renal impairment, were resolved after therapy ${ }^{(34)}$. The main factor limiting the widespread use of lipid-AmpB formulations is their high cost. Therefore, the search continues for a low-cost formulation that can be used to effectively treat leishmaniasis.

Typically, delivery using nano- or microstructured systems can result in a higher concentrations and slower release of drugs in several mammalian body organs, including the spleen, liver, and kidneys ${ }^{(35)(36)(37)(38)(39)}$. Such delivery systems for AmpB have been examined; Yang et al. ${ }^{(40)}$ formulated polymeric micelles prepared from a series of poly(ethylene glycol)-poly(lactide) (PLA) co-polymers with various polylactide chain lengths for AmpB. Shao et al. ${ }^{(41)}$ designed polymeric micelles using a formulation of 1,2-distearoyl-glycerol-3-phosphoethanolamine$\mathrm{N}$-[methoxy(polyethylene glycol)-2000] (PE-PEG)-based micelles loaded with $\mathrm{AmpB}$, and they evaluated the presence and concentration of this drug in the brains of Sprague-Dawley rats. The authors showed that this formulation was effective in penetrating the blood-brain barrier of the animals, and AmpB was found to be concentrated in the brain. Carvalho et al. ${ }^{(42)}$ developed a system containing free desoxycholate AmpB encapsulated in poly(lactic-co-glycolic acid) (PLGA) 


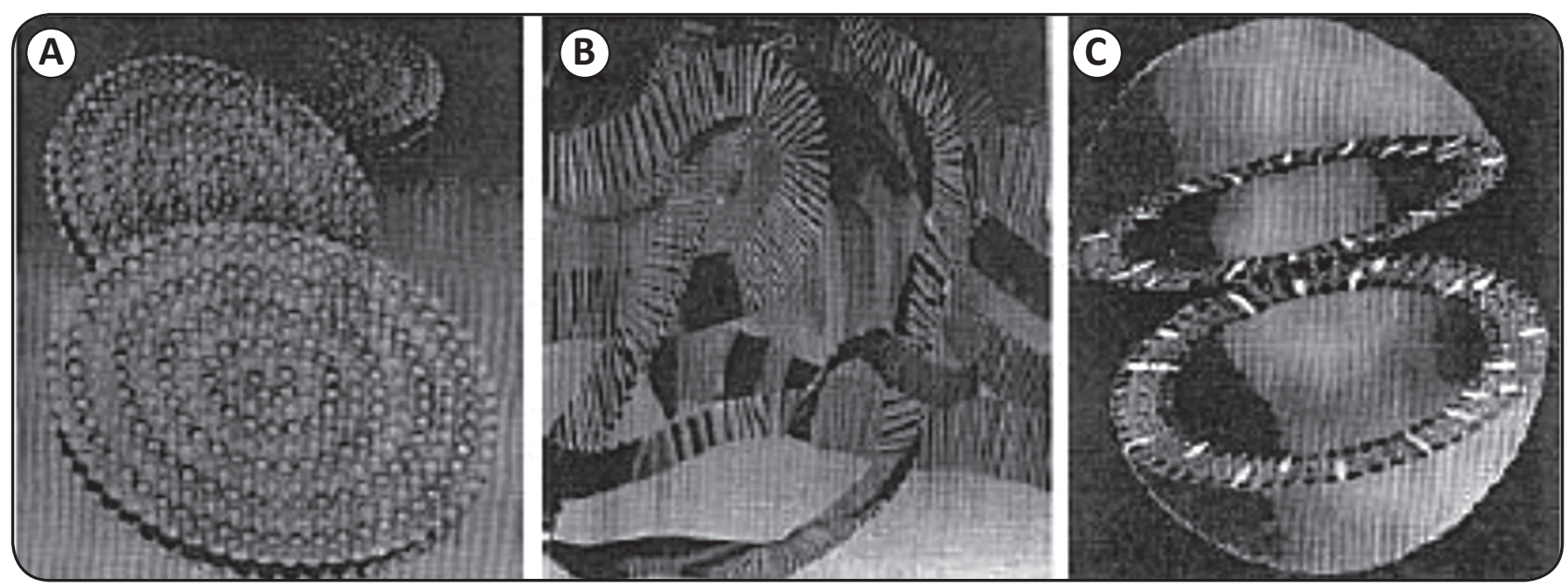

FIGURE 2 - Lipid-based formulations of AmpB. The structural characterization of three lipid-based formulations of amphotericin B are shown A: AmBisome ${ }^{\circledR}$, B: AmphocilH ${ }^{\circledR}$ and C: Abelcet ${ }^{\circledR}$.

and dimercaptosuccinic acid (DMSA), yielding PLGA-DMSA nanoparticles. These products were evaluated in the treatment of experimental cutaneous leishmaniasis in C57BL/6 mice to determine whether the nano-drug delivery system would favor a reduction in the dose frequency required to achieve the same therapeutic level as free $\mathrm{AmpB}$, thereby extending the dosing interval. The authors evaluated paw diameter measurements and the parasite loads of the animals by using a limiting dilution technique, and they found that the nano-AmpB delivery system was more effective than free $\mathrm{AmpB}$ therapy, allowing a reduction in the dose frequency required to obtain the same therapeutic level. The authors concluded that this system could favor a longer interval between doses, as expected with the development of a new nano-drug delivery system, and that it might be useful in the treatment of different pathologies, including leishmaniasis.

Silva et al. ${ }^{(43)}$ developed an oil-in-water microemulsion system (MES) to improve the solubility of AmpB. In their study, different nonionic surfactants, such as Tween ${ }^{\circledR}$ and Span ${ }^{\circledR}$, were tested for their solubilization capacity in combination with different oils. The AmpB-loaded MES was able to improve the solubility of $\mathrm{AmpB}$ up to 1,000-fold, and a portion of the $\mathrm{AmpB}$ in the system was aggregated in a drug reservoir format. Additionally, cytotoxicity studies revealed limited toxicity to macrophages, suggesting that this formulation could be a suitable carrier for AmpB that would also reduce its in vivo toxicity. Unfortunately, such examples frequently require the use of organic solvents or heating, which are undesirable steps because they can affect the integrity of macrolide substances ${ }^{(44)(45)}$. Recently, Gupta et al. ${ }^{(46)}$ developed a formulation based on the incorporation of AmpB into lipo-polymerosome (AmBL-Psome ${ }^{\circledR}$ ), and they performed in vitro (with intracellular amastigotes) and in vivo (with $L$. donovani-infected hamsters) studies comparing this delivery system with commercial Ambisome ${ }^{\circledR}$ and Fungizone ${ }^{\circledR}$. The authors showed that AmB-L-Psome ${ }^{\circledR}$ displayed lower toxicity than Fungizone ${ }^{\circledR}$ and Ambisome ${ }^{\circledR}$ along with higher antileishmanial activity.
Their results were based on the up-regulation of Th1 cytokines [tumor necrosis factor alpha (TNF- $\alpha$ ), interleukin-12 (IL-12) and interferon gamma (IFN- $\gamma$ )] and inducible nitric oxide synthase and the down-regulation of Th2 cytokines [tumor necrosis factor beta (TNF- $\beta$ ), IL-10 and IL-4], which were evaluated by quantitative messenger ribonucleic acid (mRNA) detection in infected animals using real-time reverse-transcriptasepolymerase chain reaction (RT-PCR). The authors concluded that their newly developed AmB-L-Psome ${ }^{\circledR}$ system could be a viable alternative to the less stable and more toxic commercial formulations in current use and that it could be developed as a highly efficacious drug delivery system.

In addition, Kumar et al. ${ }^{(47)}$ developed carboxy-terminated poly(D,L-lactide-co-glycolide)-block-poly(ethylene glycol) (PLGA-PEG)-encapsulated AmpB nanoparticles to increase the solubility of AmpB and to target the macrophages of infected tissues during $L$. donovani infection. Their study showed that the cytotoxicity of extracellular promastigotes when using PLGAPEG-encapsulated AmpB was significantly lower than that with free AmpB and that the inhibition of amastigote infection in the spleens of the animals was significantly higher than with free AmpB. The authors concluded that the PLGA-PEGencapsulated AmpB nanoparticles were more therapeutically effective than free AmpB in both in vitro and in vivo studies of L. donovani infection.

\section{OPTIMIZED NANOPARTICLE DELIVERY SYSTEMS FOR AMPB BASED ON CHITOSAN AND CHONDROITIN SULFATE MOLECULES}

Because of the above-mentioned problems, the development of new delivery systems for the treatment of leishmaniasis is considered important. Polyelectrolyte complexes (PECs) are formed by interactions between macromolecules bearing oppositely charged groups. The encapsulation of a drug during 
the formation of PECs has shown great promise for use as a drug delivery system ${ }^{(48)}$. Recently, PECs prepared from natural macromolecules, such as chitosan (Cs) and heparin complexes, have been formulated for use as vaccine adjuvants and have attracted attention for the development of new drug delivery systems $^{(49)}$. Studies have also investigated drug delivery systems based on Cs and chondroitin sulfate (ChS) molecules. Such reports include Ganza-Gonzales et al. ${ }^{(50)}$, Sui et al. ${ }^{(51)}$, and Huang et al. ${ }^{(52)}$, which described the use of Cs-Chs-based microcapsules for the controlled release of heparin, metoclopramide and 5 -fluorouracil, respectively. The main advantage of this method is that it allows the production of delivery systems without the need for organic solvents in their formulations ${ }^{(30)}$.

Chitosan [b-(1,4)-2-amino-2-deoxy-D-glucan], which is prepared from chitin by deacetylation, has many interesting properties, including biocompatibility, biodegradability, mucoadhesivity, and bioactivity ${ }^{(53)}$. Chitosan has an intrinsic pKa near 6.5, with a maximum net positive charge of one per residue ${ }^{(54)}$. Chondroitin sulfates are glycosaminoglycans that are present in the extracellular matrix of cartilage, and they are used for the treatment of osteoarthritis and for their anti-inflammatory activity ${ }^{(55)}$. They are also components of the dermal layer of an Food and Drug Administration (FDA)approved skin substitute ${ }^{(56)}$ and are commonly used to enhance re-epithelialization without causing scarring ${ }^{(57)}(58)$. Moreover, $\mathrm{ChS}$ molecules are similar to their endogenous homologs, which can be degraded by the colonic microflora ${ }^{(59)}$.

Recently, an optimized nanoparticle system, based on PECs using natural polymers ( $\mathrm{Cs}$ and $\mathrm{ChS}$ ) and incorporating $\mathrm{AmpB}$, was developed (Figure 3), and its antileishmanial activity was evaluated against extracellular promastigotes and intracellular amastigotes of $L$. infantum and L. amazonensis ${ }^{(30)}$. In this study, Cs nanoparticles (NQ), Cs-ChS nanoparticles (NQC), and Cs-ChS-AmpB nanoparticles (NQC-AmpB) all showed effective in vitro antileishmanial activity. In addition, a synergistic interaction between the $\mathrm{Cs}$ and $\mathrm{ChS}$ molecules was observed. Interestingly, the toxicity of the NQC-AmpB system was approximately 10 times less than that of free AmpB. In addition, the selectivity index (SI), an indicator of the selectivity of drugs tested in in vivo models ${ }^{(60)}$, was increased by 10 -fold when AmpB was added to the proposed nanodelivery system. Because the nanoparticle formulations did not cause any hemolysis of human red blood cells ${ }^{(31)}$ (Table 1), the study suggested that the NQCAmpB nanoparticles could be used as an AmpB delivery system to be evaluated in in vivo models against Leishmania spp. infections.

Another study was performed using NQC-AmpB nanoparticles to evaluate the ex vivo biodistribution profile of this delivery system in murine models. The compounds were radiolabeled with ${ }^{99 \mathrm{~m}}$ Tecnecium, and high uptake of NQCAmpB nanoparticles in the livers and spleens of naive BALB/c mice was observed by scintigraphic images up to 24 hours after administration $^{(61)}$. These findings corroborated other studies evaluating nanostructured systems, such as polymeric micelles ${ }^{(62)}$ and gold nanoparticles ${ }^{(63)}$, in which higher concentrations of the products were also found in these organs. Because the spleen is considered a systemic organ for the transit and homing of T cells

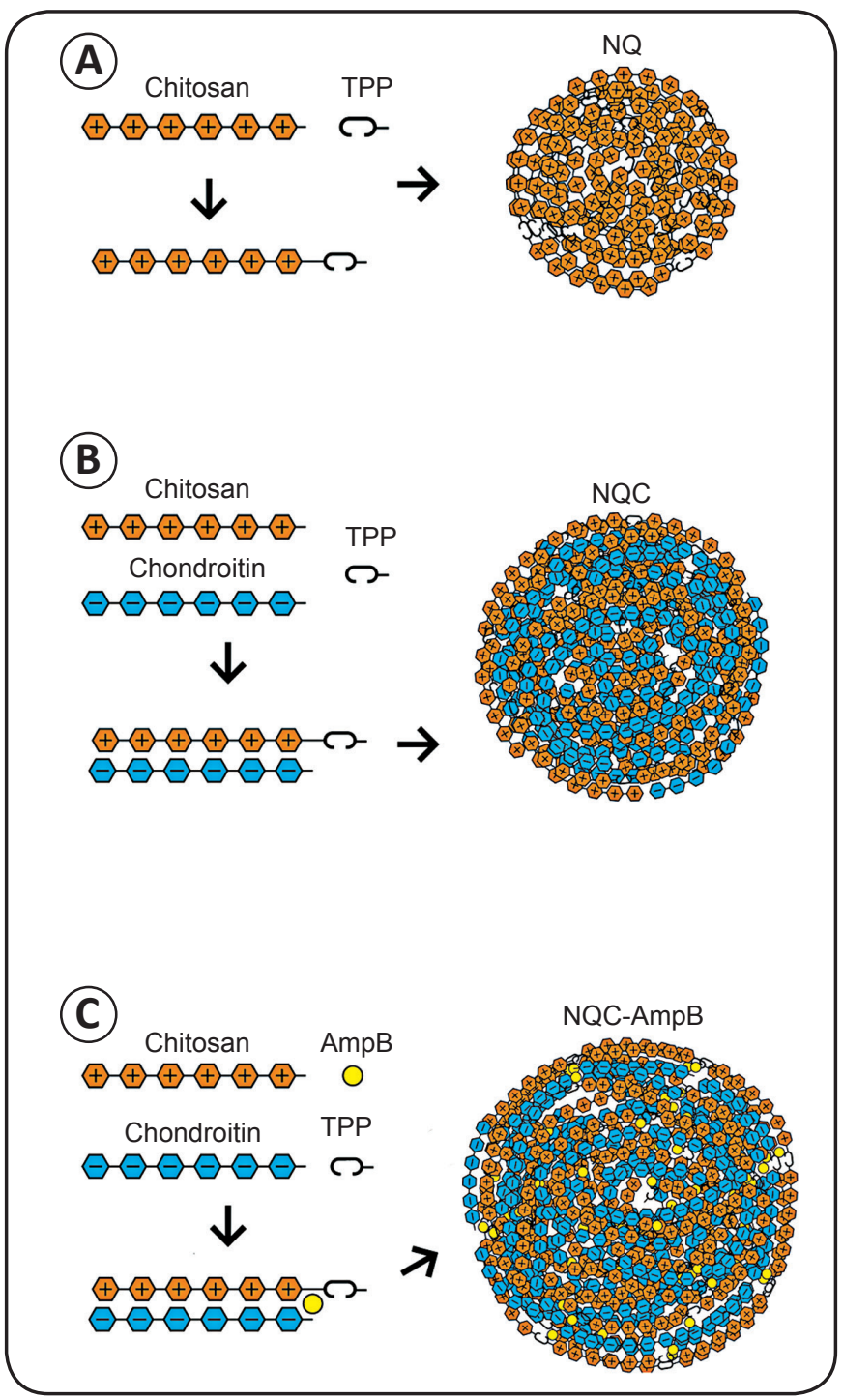

FIGURE 3 - Preparation of NQC-AmpB nanoparticles. The diagram shows models of the engineered nanoparticles, namely (A) NQ, (B) NQC, and (C) NQC-AmpB nanoparticles. The NQ nanoparticles were formulated by the PEC technique using $\mathrm{Cs}$ and TPP. The NQC nanoparticles were formulated by PEC using Cs, ChS and TPP, and the NQC-AmpB nanoparticles were formulated by PEC using $\mathrm{Cs}, \mathrm{ChS}$, and TPP, and AmpB. NQ: chitosan nanoparticle; TPP: sodium triphosphate; NQC-AmpB: chitosan-chondroitin sulfate nanoparticle-amphotericin B; AmpB: amphotericin B; NQC: chitosan-chondroitin sulfate nanoparticle; PEC: polyelectrolyte complexation; Cs: chitosan; $\mathrm{ChS}$ : chondroitin sulfate.

in mammal hosts, and because it is an important site of infection for Leishmania spp. parasites ${ }^{(64)}$, one may speculate that the greater presence of the NQC-AmpB nanoparticles in the spleen might be adequate for the treatment of leishmaniasis. Remarkably, ChS has been detected in many tissues and host cells ${ }^{(65)}$, and it has been reported to biologically interact with important molecules and to regulate their functions. It was reported that the Th1-promoting and Th2-inhibitory activities of $\mathrm{ChS}$ were associated with its binding to such adhesion molecules as L- and P-selectins, clustter of differentiation 4 (CD4) molecules, and chemokines ${ }^{(6)}$. The immune-stimulatory activity of $\mathrm{ChS}$ 
TABLE 1 - Antileishmanial activity, cytotoxicity, hemolytic activity and selectivity index of Cs, ChS, NQ, NQC, NQC-AmpB nanoparticles and free AmpB.

Inhibitory activity $(\mu \mathrm{g} / \mathrm{mL})$

\begin{tabular}{|c|c|c|c|c|c|c|}
\hline \multirow[b]{2}{*}{ Compounds and formulations } & \multirow{2}{*}{$\begin{array}{l}\text { L. amazonensis } \\
\qquad\left(\mathrm{IC}_{50}\right)^{\mathrm{a}}\end{array}$} & \multirow{2}{*}{$\begin{array}{l}\text { L. infantum } \\
\qquad\left(\mathrm{IC}_{50}\right) \\
\end{array}$} & \multirow{2}{*}{$\begin{array}{l}\text { Murine macrophages } \\
\qquad\left(\mathrm{CC}_{50}\right)^{\mathrm{b}}\end{array}$} & \multirow{2}{*}{$\begin{array}{l}\text { Red blood cells } \\
\qquad\left(\mathrm{RBC}_{50}\right)^{\mathrm{c}}\end{array}$} & \multicolumn{2}{|c|}{$\begin{array}{l}\text { Selectivity index } \\
\left(\mathrm{SI}: \mathrm{CC}_{50} / \mathrm{IC}_{50}\right)^{\mathrm{d}}\end{array}$} \\
\hline & & & & & L. amazonensis & L. infantum \\
\hline $\mathrm{ChS}$ & $65.99 \pm 5.60$ & $71.00 \pm 10.00$ & $1,349.00 \pm 142.00$ & $697.00 \pm 106.00$ & 20.43 & 19.00 \\
\hline NQ & $51.86 \pm 1.74$ & $45.64 \pm 5.51$ & $831.19 \pm 13.22$ & $237.83 \pm 25.50$ & 16.03 & 18.21 \\
\hline NQC-AmpB & $0.09 \pm 0.01$ & $0.11 \pm 0.07$ & $8.92 \pm 0.18$ & $239.65 \pm 32.46$ & 94.93 & 83.39 \\
\hline AmpB & $0.10 \pm 0.02$ & $0.09 \pm 0.01$ & $0.78 \pm 0.21$ & $12.21 \pm 2.70$ & 7.80 & 8.67 \\
\hline
\end{tabular}

may be due to its binding to L-selectins expressed on the $\mathrm{T}$ cell surface ${ }^{(67)}$. In this sense, the cellular response induced by $\mathrm{Cs}$ in an infected spleen might help the host immune system to eliminate intracellular amastigotes. In addition, a toxicological analysis was performed on the livers of animals that received NQC-AmpB nanoparticles. A significant increase in hepatocyteassociated serum enzymes was observed in animals treated with free AmpB, resulting in significant elevation of the levels of gamma-glutamyltransferase (GGT), aspartate aminotransferase (AST), and alanine aminotransferase (ALT) enzymes. Meanwhile, animals that received NQC-AmpB did not show any enzymatic alterations ${ }^{(61)}$. These results demonstrated a decrease in hepatic toxicity when AmpB was administered using the NQC system ${ }^{(61)}$.

Nephrotoxicity is perhaps the most-described adverse event associated with $\mathrm{AmpB}^{(68)}$. The pathophysiology and pharmacology of this activity are well-documented, and the proposed mechanism has been described as multifactorial ${ }^{(69)(70)}$. Early studies proposed renal tubular toxicity and afferent arteriolar vasoconstriction as possible physiological factors related to AmpB-induced nephrotoxicity, but the underlying mechanistic explanation for injury remains poorly understood ${ }^{(71)}$. Inflammation plays a central role in the pathogenesis of drug-induced kidney injury. Inflammation is mediated by the expression of cytokines upon the activation of Toll-like receptors (TLRs) located on the renal parenchymal and tubule-interstitial cells. The net inflammatory response entails a coordinated balance between pro-inflammatory and anti-inflammatory mediators; a relative exuberance of the former predisposes tissue to injury. The pro-inflammatory nature of AmpB has been characterized, and it is believed to be mediated by the engagement of TLR2 and TLR4, which results in the activation of NF-kB and the production of cytokines ${ }^{(72)}$. In this context, Chai et al. ${ }^{(73)}$ demonstrated that, among patients receiving AmpB therapy, temporal trends in pro-inflammatory cytokine levels had greater clinical significance than their absolute levels. As new light is shed on the mechanisms of AmpB-induced nephrotoxicity via the signaling pathways that mediate tissue inflammation, an important goal is to develop novel management and/or delivery strategies that minimize the adverse effects of this time-tested antifungal agent. One such measure might be the use of $\mathrm{N}$-acetylcysteine, which, in accord with its utility in contrast-induced nephropathy, also has anti-inflammatory and therapeutic properties ${ }^{(74)}$. In addition, delivery systems based on ChS molecules may be useful, as these molecules also possess anti-inflammatory activity and may reduce the nephrotoxicity caused by free AmpB.

Ribeiro et al. ${ }^{(61)}$ showed that the levels of urea in blood, as well as serum creatinine levels, were significantly higher in animals treated with free AmpB than in those treated with NQC-AmpB nanoparticles. The alterations detected in the kidneys of AmpBtreated animals by microscopic observation were in accord with those described in previous studies ${ }^{(75)}$. In contrast, animals treated with NQC-AmpB showed no pathological alterations in this tissue. It may therefore be concluded that the decrease in AmpB toxicity in the NQC-AmpB preparation relative to free AmpB might have occurred because the nanoparticle-encapsulated AmpB did not interact with the cell membranes within the kidney tubules, thereby minimizing nephrotoxicity ${ }^{(61)}$.

Finally, the efficacy of NQC-AmpB nanoparticles for treating chronically L. amazonensis-infected BALB/c mice was evaluated in comparison to the free drug. Although both NQC-AmpB and free AmpB treatments were able to induce a significant reduction in the parasite load in the infected animals (Figure 4), the NQC-AmpB system was less toxic than free AmpB. The authors concluded that NQC-AmpB nanoparticles may be used as an alternative AmpB delivery system that maintains the high activity of this drug against Leishmania spp. but significantly reduces its toxicity relative to that of the free $\mathrm{drug}^{(61)}$. 


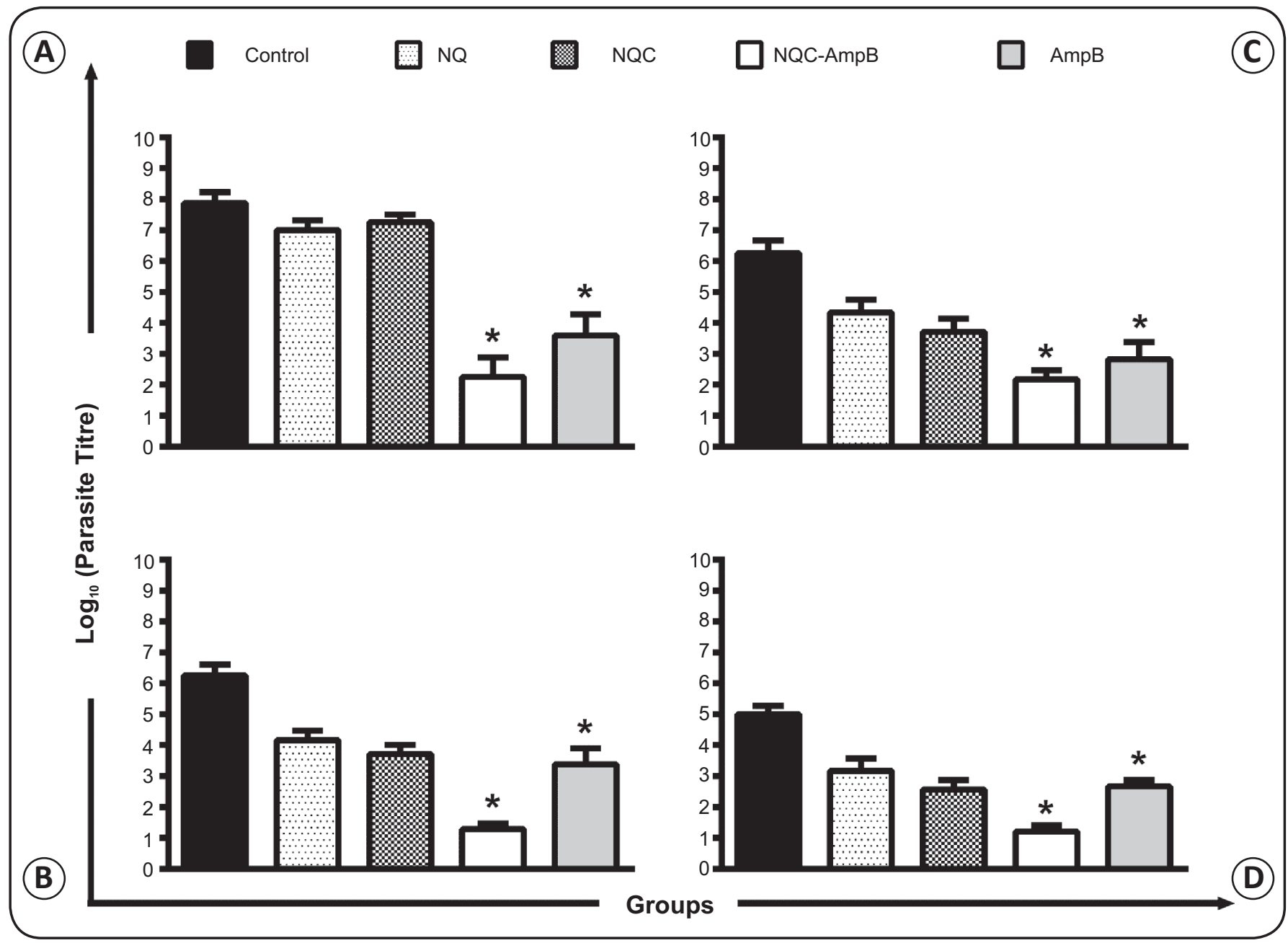

FIGURE 4 - Protective efficacy of the chitosan-chondroitin sulfate nanoparticle-amphotericin B (NQC-AmpB) nanoparticles against Leishmania amazonensis infection. BALB/c mice were subcutaneously infected with $5 \times 10^{6}$ stationary-phase promastigotes of L. amazonensis (IFLA/BR/1967/PH-8), and the course of infection was monitored for 115 days. When the animals developed ulcerated lesions presenting an average diameter of 2 to $3 \mathrm{~mm}$, they were divided into groups according to lesion size. They were then treated for 10 days with intravenous injections according to one of the following regimens: I) Control group, in which mice received $100 \mu \mathrm{L}$ of saline; II) amphotericin B (AmpB) group, in which mice received $100 \mu \mathrm{L} \mathrm{of} 1 \mathrm{mg} / \mathrm{kg}$ body weight of AmpB; III) chitosan nanoparticle (NQ) group, in which mice received $100 \mu \mathrm{L}$ of chitosan $(\mathrm{Cs})$ nanoparticles, at equivalent amounts of Cs relative to the NQC-AmpB nanoparticles; IV) chitosan-chondroitin sulfate nanoparticle (NQC) group, in which mice received $100 \mu \mathrm{L}$ of empty Cs- chondroitin sulfate (ChS) nanoparticles at equal amounts of Cs-ChS relative to the NQC-AmpB nanoparticles; -NQC-AmpB group, in which mice received $100 \mu \mathrm{L}$ of AmpB-loaded-Cs-ChS nanoparticles at the dose of $1 \mathrm{mg} / \mathrm{kg}$ body weight of AmpB. The infected mice were observed for a 30-day period after the end of treatment, when the parasite burden in the infected footpads (A), spleen (B), liver (C), and draining lymph nodes (D) was evaluated by a limiting-dilution technique. Means \pm standard deviations (SDs) of the groups are shown. Data are representative of three independent experiments, which yielded similar results. *Indicates a significant difference compared to the saline group.

\section{CONCLUSIONS}

New drug identification, development and registration represent expensive and time-consuming tasks that typically cause pharmaceutical companies to neglect new strategies for the treatment of leishmaniasis. Although some drugs that treat this disease are available, their high toxicity to patients can cause interruption of the therapeutic regimen. Therefore, it is desirable to develop new delivery systems that maintain the antileishmanial activity of these drugs but reduce their toxicity. There are some delivery systems currently under development and/or in clinical trials, but they are generally costly. In contrast, nanoparticles comprised of $\mathrm{Cs}$ and $\mathrm{ChS}$ molecules could, at least in part, solve this problem; as such nanoparticles consist of two known and commercially available products. While there is presently no estimated value for such nanoparticles, there is a real possibility that these formulations will have a lower cost than other available nano- and microstructured formulations.

\section{CONFLICT OF INTEREST}

The authors declare that there is no conflict of interest. 


\section{REFERENCES}

1. Desjeux P. Leishmaniasis: Current situation and new perspectives. Comp Immunol Microbiol Infect Dis 2004; 27:305-318.

2. World Health Organization. Control of the leishmaniases. World Health Organ Tech Rep Ser 2010; 949:22-26.

3. Alvar J, Vélez ID, Bern C, Herrero M, Desjeux P, Cano J, et al. Leishmaniasis worldwide and global estimates of its incidence. PLoS One 2012; 7:e35671.

4. Guerin PJ, Olliaro P, Sundar S, Boelaert M, Croft SL, Desjeux P, et al. Personal view visceral leishmaniasis: current status of control, diagnosis, and treatment, and a proposed research and development agenda Personal view. Lancet 2002; 2:494-501.

5. Chappuis F, Sundar S, Hailu A, Ghalib H, Rijal S, Peeling RW, et al. Visceral leishmaniasis: what are the needs for diagnosis, treatment and control? Nat Rev Microbiol 2007; 5:873-882.

6. Marinho DS, Casas CNPR, Pereira CCDA, Leite IC Health economic evaluations of visceral leishmaniasis treatments: a systematic review. PLoS Negl Trop Dis 2015; 9:e0003527.

7. Alvar J, Aparicio P, Aseffa A, Den Boer M, Cañavate C, Dedet JP, et al. The relationship between leishmaniasis and AIDS: The second 10 years. Clin Microbiol Rev 2008; 21:334-359.

8. Shafiei R, Mohebali M, Akhoundi B, Galian MS, Kalantar F, Ashkan S, et al. Emergence of co-infection of visceral leishmaniasis in HIV-positive patients in northeast Iran: A preliminary study. Travel Med Infect Dis 2014; 12:173-178.

9. Lainson R, Shaw JJ. New World leishmaniasis. In: Cox FEG, Wakelin D, Gillespie SH, Despommier DD, editors. Topley \& Wilson's Microbiology and Microbial Infections: parasitology. $10^{\text {th }}$ ed. London: Hodder Arnold ASM Press; 2005. p. 313-349.

10. Gramiccia M, Gradoni L. The current status of zoonotic leishmaniases and approaches to disease control. Int J Parasitol 2005; 35:1169-1180.

11. Apa H, Devrim I, Bayram N, Deveci R, Demir-Özek G, Carti ÖU. Liposomal amphotericin B versus pentavalent antimony salts for visceral Leishmania in children. Turk J Pediatr 2013; 55:378-383.

12. Michel G, Pomares C, Ferrua B, Marty P. Importance of worldwide asymptomatic carriers of Leishmania infantum (L. chagasi) in human. Acta Trop 2011; 119:69-75.

13. Tavares CAP, Fernandes AP, Melo MN. Molecular diagnosis of leishmaniasis. Expert Rev Mol Diagn 2003; 3:657-667.

14. Srividya G, Kulshrestha A, Singh R, Salotra P. Diagnosis of visceral leishmaniasis: Developments over the last decade. Parasitol Res 2012; 110:1065-1078.

15. Chatzis MK, Leontides L, Athanasiou LV, Papadopoulos E, Kasabalis D, Mylonakis M, et al. Evaluation of indirect immunofluorescence antibody test and enzyme-linked immunosorbent assay for the diagnosis of infection by Leishmania infantum in clinically normal and sick cats. PubMed Commons. Exp Parasitol 2014; 147:54-59.

16. Sundar S, Chakravarty J. Antimony toxicity. Int J Environ Res Public Health 2010; 7:4267-4277.

17. Wortmann G, Miller RS, Oster C, Jackson J, Aronson N. A randomized, double-blind study of the efficacy of a 10- or 20-day course of sodium stibogluconate for treatment of cutaneous leishmaniasis in United States military personnel. Clin Infect Dis 2002; 35:261-267.

18. Romero GAS, Guerra MVF, Paes MG, Macêdo VO. Comparison of cutaneous leishmaniasis due to Leishmania (Viannia) braziliensis and $L$. $(V$.) guyanensis in Brazil: therapeutic response to meglumine antimoniate. Am J Trop Med Hyg 2001; 65:456-465.

19. Sundar S. Drug resistance in Indian visceral leishmaniasis. Trop Med Int Heal 2001; 6:849-854.

20. Croft SL, Coombs GH. Leishmaniasis: current chemotherapy and recent advances in the search for novel drugs. Trends Parasitol 2003; 19:502-508.
21. Marsden PD. Pentavalent antimonials: old drugs for new diseases. Rev Soc Bras Med Trop 1985; 18:187-198.

22. Barral-Netto M, Barral A, Brodskyn C, Carvalho EM, Reed SG. Cytotoxicity in human mucosal and cutaneous leishmaniasis. Parasite Immunol 1995; 17:21-28.

23. Bhattacharya SK, Jha TK, Sundar S, Thakur CP, Engel J, Sindermann H, et al. Efficacy and tolerability of miltefosine for childhood visceral leishmaniasis in India. Clin Infect Dis 2004; $38: 217-221$.

24. Vyas SP, Gupta S. Optimizing efficacy of amphotericin B through nanomodification. Int J Nanomedicine 2006; 1:417-432.

25. Bleicher KH, Böhm H-J, Müller K, Alanine AI. Hit and lead generation: beyond high-throughput screening. Nat Rev Drug Discov 2003; 2:369-378.

26. Hughes JP, Rees SS, Kalindjian SB, Philpott KL. Principles of early drug discovery. Br J Pharmacol 2011; 162:1239-1249.

27. Seifert K. Structures, targets and recent approaches in antileishmanial drug discovery and development. Open Med Chem J 2011; 5:31-39.

28. Morais-Teixeira E, Gallupo MK, Rodrigues LF, Romanha ALJ, Rabello A. In vitro interaction between paromomycin sulphate and four drugs with leishmanicidal activity against three New World Leishmania species. J Antimicrob Chemother 2014; 69: 150-154.

29. Annaloro C, Olivares C, Usardi P, Onida F, Della Volpe A, Tagliaferri E, et al. Retrospective evaluation of amphotericin B deoxycholate toxicity in a single centre series of haematopoietic stem cell transplantation recipients. J Antimicrob Chemother 2009; 63:625-626.

30. Ribeiro TG, Chávez-Fumagall MA, Valadares DG, França JR, Rodrigues LB, Duarte MC, et al. Novel targeting using nanoparticles: An approach to the development of an effective anti-leishmanial drug-delivery system. Int J Nanomedicine 2014; 9:877-890

31. Bern C, Adler-Moore J, Berenguer J, Boelaert M, Boer M, Davidson RN, et al. Liposomal amphotericin B for the treatment of visceral leishmaniasis. Clin Infect Dis 2006; 43:917-924.

32. Rosenthal E, Delaunay P, Jeandel PY, Haas H, Pomares-Estran C, Marty P. Le traitement de la leishmaniose viscérale en Europe en 2009. Place de l'amphotéricine B liposomale. Med Mal Infect 2009; 39:741-744.

33. Solomon M, Pavlotsky F, Leshem E, Ephros M, Trau H, Schwartz E. Liposomal amphotericin B treatment of cutaneous leishmaniasis due to Leishmania tropica. J Eur Acad Dermatology Venereol 2011; 25:973-977.

34. Sundar S, Chakravarty J. Leishmaniasis: an update of current pharmacotherapy. Expert Opin Pharmacother 2013; 14:53-63.

35. Saxena S, Ghosh PC. Biodistribution of amphotericin B when delivered through cholesterol hemisuccinate vesicles in normal and A. fumigatus infected mice. Pharm Res 2000; 17:1236-1242.

36. Townsend RW, Zutshi A, Bekersky I. Biodistribution of 4-[(14) C]cholesterol-AmBisome following a single intravenous administration to rats. Drug Metab Dispos 2001; 29:681-685.

37. Agrawal AK, Agrawal A, Pal A, Guru PY, Gupta CM. Superior chemotherapeutic efficacy of amphotericin B in tuftsin-bearing liposomes against Leishmania donovani infection in hamsters. J Drug Target 2002; 10:41-45.

38. Bekersky I, Fielding RM, Dressler DE, Lee W, Buell DN, Walsh TJ, et al. Pharmacokinetics, Excretion, and mass balance of liposomal amphotericin B (Ambisome) and amphotericin B deoxycholate in humans. Antimicrob Agents Chemother 2002; 46:828-833.

39. Gershkovich P, Wasan EK, Lin M, Sivak O, Leon CG, Clement JG, et al. Pharmacokinetics and biodistribution of amphotericin $\mathrm{B}$ in rats following oral administration in a novel lipid-based formulation. J Antimicrob Chemother 2009; 64:101-108. 
40. Yang ZL, Li XR, Yang KW, Liu Y. Amphotericin B-loaded poly(ethylene glycol)-poly(lactide) micelles: preparation, freezedrying, and in vitro release. J Biomed Mater Res - Part A 2008; 85:539-546.

41. Shao K, Huang R, Li J, Han L, Ye L, Lou J, et al. Angiopep-2 modified PE-PEG based polymeric micelles for amphotericin B delivery targeted to the brain. J Control Release 2010; 147:118-126.

42. Carvalho RF, Ribeiro IF, Miranda-Vilela AL, Souza-Filho J, Martins OP, Oliveira CSD, et al. Leishmanicidal activity of amphotericin B encapsulated in PLGA-DMSA nanoparticles to treat cutaneous leishmaniasis in C57BL/6 mice. Exp Parasitol 2013; 135:217-222.

43. Silva AE, Barratt G, Cheŕon M, Egito EST. Development of oilin-water microemulsions for the oral delivery of amphotericin B. Int J Pharm 2013; 454:641-648.

44. Kreuter J. Possibilities of using nanoparticles as carriers for drugs and vaccines. J Microencapsul 1988; 5:1155:115-127.

45. Quintanar-Guerrero D, Allémann E, Fessi H, Doelker E. Preparation techniques and mechanisms of formation of biodegradable nanoparticles from preformed polymers. Drug Dev Ind Pharm 1998; 24:1113-1128.

46. Kumar R, Sahoo GC, Pandey K, Das VNR, Das P. Study the effects of PLGA-PEG encapsulated amphotericin B nanoparticle drug delivery system against Leishmania donovani. Drug Deliv 2014; Ahead of Print. doi:10.3109/10717544.2014.891271.

47. Gupta PK, Jaiswal AK, Kumar V, Verma A, Dwivedi P, Dube A, et al. Covalent functionalized self-assembled lipo-polymerosome bearing amphotericin $\mathrm{B}$ for better management of leishmaniasis and its toxicity evaluation. Mol Pharm 2014; 11:951-963.

48. Janes KA, Fresneau MP, Marazuela A, Fabra A, Alonso MJ. Chitosan nanoparticles as delivery system for doxorubicin. J Control Rel 2001; 73:255-267.

49. John AL, Chan CY, Staats HF, Leong KW, Abraham SN. Synthetic mast-cell granules as adjuvants to promote and polarize immunity in lymph nodes. Nat Mater 2012; 11:250-257.

50. Ganza-González A, Anguiano-Igea S, Otero-Espinar FJ, Blanco Méndez J. Chitosan and chondroitin microspheres for oraladministration controlled release of metoclopramide. Eur J Pharm Biopharm 1999; 48:149-155.

51. Sui W, Huang L, Wang J, Bo Q. Preparation and properties of chitosan chondroitin sulfate complex microcapsules. Colloids Surfaces B Biointerfaces 2008; 65:69-73.

52. Huang L, Sui W, Wang Y, Jiao Q. Preparation of chitosan/ chondroitin sulfate complex microcapsules and application in controlled release of 5-fluorouracil. Carbohydr Polym 2010; 80:168-173.

53. Bhattarai N, Gunn J, Zhang M. Chitosan-based hydrogels for controlled, localized drug delivery. Adv Drug Deliv Rev 2010; 62:83-99.

54. Thongngam $\mathrm{M}, \mathrm{McClements} \mathrm{DJ}$. Influence of $\mathrm{pH}$, ionic strength, and temperature on self-association and interactions of sodium dodecyl sulfate in the absence and presence of chitosan. Langmuir $2005 ; 21: 79-86$

55. Iovu M, Dumais G, Souich P. Anti-inflammatory activity of chondroitin sulfate. Osteoarthr Cartil 2008; 16:14-18.

56. Kirker KR, Luo Y, Nielson JH, Shelby J, Prestwich GD. Glycosaminoglycan hydrogel films as bio-interactive dressings for wound healing. Biomaterials 2002; 23:3661-3671.

57. Kirker KR, Luo Y, Nielson JH, Shelby J, Prestwich GD. Glycosaminoglycan hydrogel films as bio-interactive dressings for wound healing. Biomaterials 2002; 23:3661-3671.

58. Wang LF, Wang JM, Chiang YL. Insolubilization of sodium chondroitin sulfate by forming a semi-interpenetrating polymer network with acrylic acid: A potential carrier for colon-specific drug delivery. J Appl Polym Sci 2002; 85:114-122.
59. Sintov A, Di-Capua N, Rubinstein A. Cross-linked chondroitin sulphate: Characterization for drug delivery purposes. Biomaterials 1995; 16:473-478.

60. Nwaka S, Hudson A. Innovative lead discovery strategies for tropical diseases. Nat Rev Drug Discov 2006; 5:941-955.

61. Ribeiro TG, Franca JR, Fuscaldi LL, Santos ML, Duarte MC, Lage PS, et al. An optimized nanoparticle delivery system based on chitosan and chondroitin sulfate molecules reduces the toxicity of amphotericin B and is effective in treating tegumentary leishmaniasis. Int J Nan 2014; 9:5341-5353.

62. Koide H, Asai T, Hatanaka K, Urakami T, Ishii T, Kenjo E, et al. Particle size-dependent triggering of accelerated blood clearance phenomenon. Int J Pharm 2008; 362:197-200.

63. Jong L, Moreau X, Thiéry A, Godeau G, Grinstaff MW, Barthélémy P. Amphiphilic copolymer for delivery of xenobiotics: in vivo studies in a freshwater invertebrate, a mesostominae flatworm. Bioconjug Chem 2008; 19:891-898.

64. Oliveira DM, Costa MAF, Chavez-Fumagalli MA, Valadares DG, Duarte MC, Costa LE, et al. Evaluation of parasitological and immunological parameters of Leishmania chagasi infection in $\mathrm{BALB} / \mathrm{c}$ mice using different doses and routes of inoculation of parasites. Parasitol Res 2012; 110:1277-1285.

65. Ohhashi Y, Hasumi F, Mori Y. Comparative study on glycosaminoglycans synthesized in peripheral and peritoneal polymorphonuclear leucocytes from guinea pigs. Biochem J 1984; 217:199-207.

66. Stevens RL, Fox CC, Lichtenstein LM, Austen KF. Identification of chondroitin sulfate E proteoglycans and heparin proteoglycans in the secretory granules of human lung mast cells. Proc Natl Acad Sci USA 1988; 85:2284-2287.

67. Akiyama H, Sakai S, Linhardt RJ, Goda Y, Toida T, Maitani T. Chondroitin sulphate structure affects its immunological activities on murine splenocytes sensitized with ovalbumin. Biochem J 2004; 382:269-278.

68. Martinez-Pomares L, Hanitsch LG, Stillion R, Keshav S, Gordon $\mathrm{S}$. Expression of mannose receptor and ligands for its cysteinerich domain in venous sinuses of human spleen. Lab Invest 2005; 85:1238-1249.

69. Bagnis CI, Deray G. Amphotericin B Nephotoxicity. Saudi J Kidney Dis Transplant 2002; 13:481-491.

70. Takahisatyano Y, Yoshinori I, Eiko K, Asuka M, Nobuaki E, Motohiro N, et al. Amphotericin B-induced renal tubular cell injury is mediated by $\mathrm{Na}^{+}$influx through ion-permeable pores and subsequent activation of mitogen-activated protein kinases and elevation of intracellular $\mathrm{Ca}^{2+}$ concentration. Antimicrob Agents Chemother 2009; 53:1420-1426.

71. Sau K, Mambula SS, Latz E, Henneke P, Golenbock DT, Levitz $\mathrm{SM}$. The antifungal drug amphotericin B promotes inflammatory cytokine release by a Toll-like receptor- and CD14-dependent mechanism. J Biol Chem 2003; 278: 37561-37568.

72. Bellocchio S, Gaziano R, Bozza S, Rossi G, Montagnoli C, Perruccio K, et al. Liposomal amphotericin B activates antifungal resistance with reduced toxicity by diverting Toll-like receptor signalling from TLR-2 to TLR-4. J Antimicrob Chemother 2005; 55: 214-222.

73. Chai LY, Netea MG, Tai BC, Khin LW, Vonk AG, Teo BW, et al. An elevated pro-inflamatory cytokine response is linked to development of amphotericin B-induced nephrotoxicity. J Antimicrob Chemother. 2013; 68:1655-1659.

74. Odabasi Z, Karaalp A, Cermik H, Mohr J, Tigen ET, Koc M. Reduction of amphotericin B-induced renal tubular apoptosis by $\mathrm{N}$-acetylcysteine. Antimicrob Agents Chemother 2009; 53: 3100-3102.

75. Patel GP, Crank CW, Leikin JB. An Evaluation of hepatotoxicity and nephrotoxicity of liposomal amphotericin B (L-AMB). J Med Toxicol 2011; 7:12-15. 